\title{
Research on new energy controllable rectifier based on DSP ZHANG Ruo-bing
}

\author{
(Hunan Railway Professional Technology College, ZhuZhou Hunan 412001)
}

\author{
Keywords: Energy, Rectifier, DSP
}

\begin{abstract}
Three-phase PWM rectifier of New Energy based on DSP has been put forward by the disadvantages of random fluctuation about New Energy and the deficiency of the traditional rectifier . the Mathematic model of three-phase PWM rectifier in the d-q rotation frames is analyzed at first, to decouple the three-phase system, the current feed-forward compensation based on synchronous rotation frames was used, the current model decoupled was equal to one-order system and realized the separated control on the active and inactive current . The Space Vector Pulse Width Modulation ( SVPWM ) scheme is applied into the control of three-phase PWM rectifier and decoupling control has been realized by DSP TMS320F28027. The experimental results showed the feasibility of the design scheme of the system.
\end{abstract}

\section{Introduction}

After entering in twenty-first Century, the trend of worldwide energy shortage is more and more obvious, the energy crisis has triggered a series of global problems, the solution is only one, that is to develop new forms of energy and new energy saving technology combining the basis to establish a new type of clean, safe and sustainable energy system. New energy because of its no pollution, renewable, widely distributed characteristics such as being more and more accepted and recognized, but there are also disadvantages: long random energy fluctuations when fluctuations (diurnal or seasonal cycles), short-term fluctuations (hour level) and instantaneous fluctuation (seconds or less), with intermittent power supply instability, so how to implement the rectification of new energy is still a big problem: the traditional thyristor phase controlled rectifier has defects of low power factor and harmonic; although the diode rectifier input current fundamental no lag, the displacement factor is close to 1, but the harmonic current is great. According to the above description of the problem, we propose TMS320F28027 new energy controlled rectifier and control technology of the design and implementation scheme based on solving the problems of the big harmonic content, not energy feedback and low power factor rectifier circuit in the traditional sense, as a new energy conversion and control technology, the breakthrough of the technical warning.

\section{Control strategy of three phase VSR}

With the wide application of PWM rectifier in high performance power electronic devices, in order to achieve higher performance requirements, the control strategies have been launched, mainly around the implementation of unit power factor, reduce switching loss and improve the dynamic response and efficiency of several aspects of the system. In the design of control system of three-phase VSR, double closed-loop control system with voltage loop and current loop and outer voltage loop is $U_{d}$ DC voltage control of three-phase VSR, and given the active current of $i_{d}^{*}$; the role of the current network side current tracking command current, the power factor of the unit or controlled. As shown in Fig.1. 


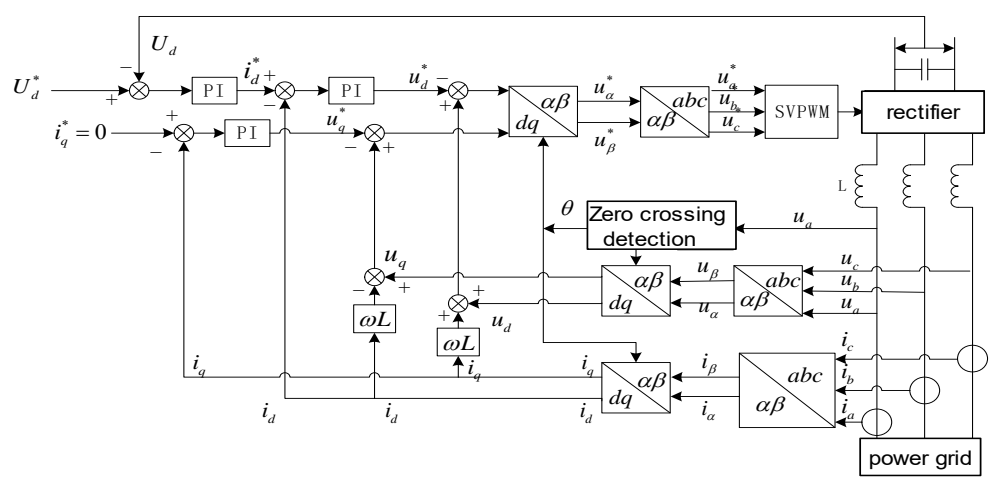

Fig. 1 control principle of three-phase VSR

In the three-phase VSR control system, the double closed loop control of current inner loop and voltage outer loop has been achieved.

\section{3 system hardware design}

\subsection{Main circuit structure and action analysis of three phase PWM rectifier}

The main circuit topology of three-phase PWM rectifier circuit as shown in Fig.2, the left half of the three-phase PWM rectifier, the right part is the zero voltage switching circuit (ZVS), and the switch devices are connected in parallel with the buffer capacitor.

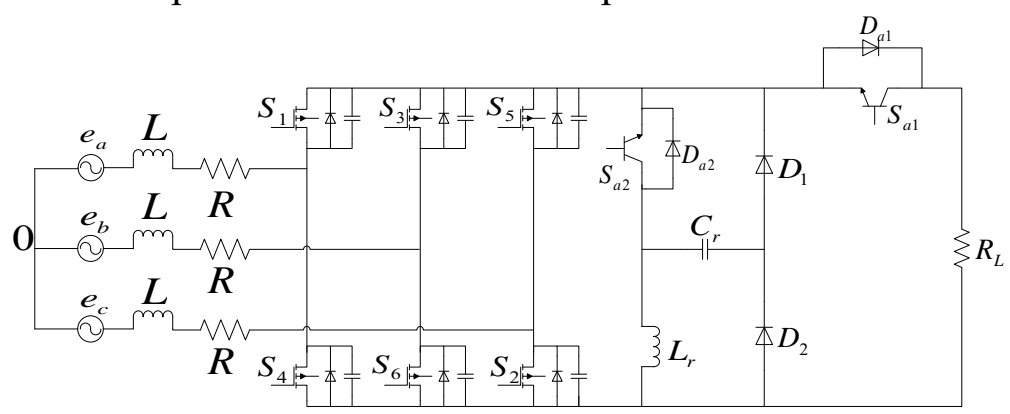

Fig.2 Three phase PWM rectifier topology

Since the switching frequency rectifier is much higher than the frequency of the grid, so in a switching period can be considered as the input current and output current of the rectifier is constant, which can be used to represent the constant current source and the input current and output current, so it can be used Fig. 3as the equivalent of Fig. 2.

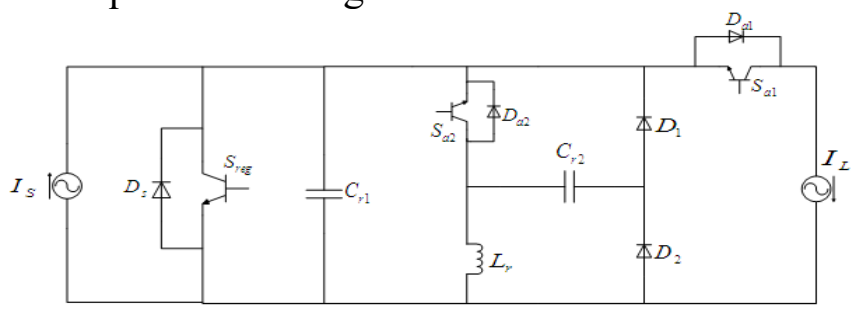

Fig.3 Equivalent diagram of three-phase PWM rectifier

In Fig.3, $S_{r e g} 、 D_{s} 、 C_{r 1}$ respectively, on behalf of the rectifier power switch, freewheeling diode and snubber capacitor, Because the power switching device of the upper and the lower bridge arm of the three-phase rectifier bridge is always communicated with one side, So, $C_{r 1}=3 C_{s}$ 。 The soft switching section consists of two switching devices $S_{a 1}$ and $S_{a 2}$, Two diodes and $D_{1}$ and $D_{2}$, Resonant inductor $L_{r}$ and resonant capacitor $C_{r 1} 、 C_{r 2}, C_{r 1}$ is the main resonant capacitor in the soft switching structure, $C_{r 2}$ is an auxiliary capacitor used to reduce the resonant inductor $L_{r}$ current reverse $i_{L r}$. During the main resonant capacitor $C_{r 1}$ is zero, the switching device of three-phase bridge can be operated to achieve zero voltage operation, which greatly reduces the power consumption. 


\subsection{Design of driving circuit for main switch device}

Provided between the main circuit and control circuit interface circuit, it can according to the control requirements of the switching device applied to open or turn off signal, and the power electronic devices work in the switch state ideal, shorten switching time, reduce switching loss. In addition, some protection measures are often located in the drive circuit, or through the driver circuit.

The design of the full control device using the MOSFET, the opening of the gate voltage can not exceed the range of $12 \mathrm{~V} \sim 20 \mathrm{~V}$, when the gate voltage is 0 , in the cut-off state. We choose HCPL3180 to build the MOSFET driver circuit, the circuit diagram shown in Fig. 4.

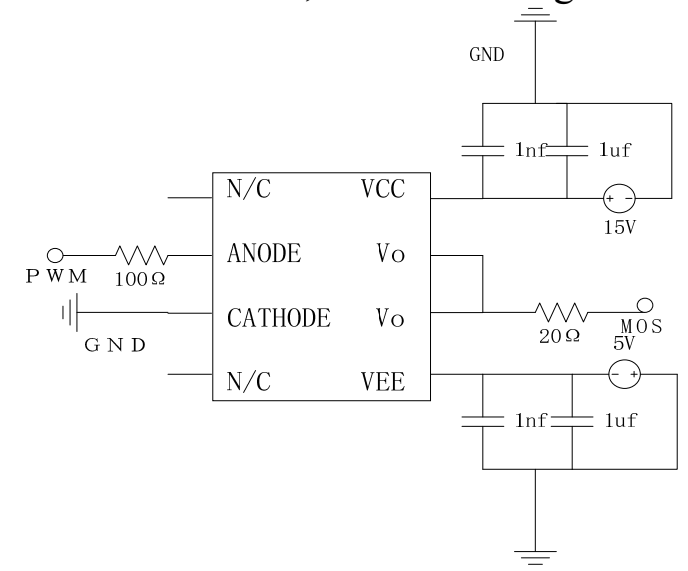

Fig.4 MOSFET drive circuit

Drive HCPL3180 high speed optocoupler AVAGO, DSP and electrical parts (MOSFET) isolated in order to protect the DSP, output current up to 2A, the better the advantages of HCPL3180 input side does not need power supply, HCPL3180 input drive diode minimum current 10mA, maximum current $16 \mathrm{~mA}$, voltage $1.5 \mathrm{~V}$, so the input current limiting resistor of $100 \Omega$.

\subsection{Design of voltage and current sampling circuit}

The current and voltage are detected by Holzer sensor, because the output voltage signal, so the current and voltage signal processing can use the same structure of the conditioning circuit. Sample conditioning circuit shown in Fig.5.

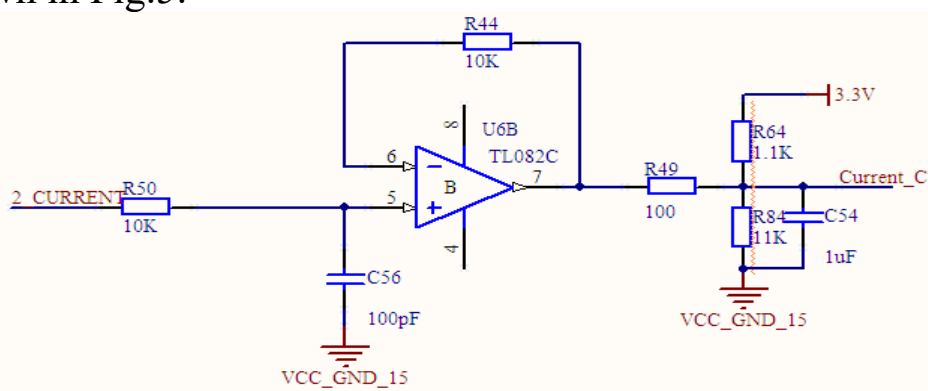

Fig.5 Sample conditioning circuit

In the design of the rectifier, the AC current signal and the voltage signal of the load side are needed to be sampled. Sampling of the current, the use of L07P020D15 Holzer current sensor; voltage sampling, using the BJHVS5-25A Holzer voltage sensor. Whether Holzer or Holzer as the voltage sensor, current sensor, its output was weak current signal into a voltage signal through the resistor R50 through the resistor, capacitor component of the filter and the phase ratio of the measured signal conditioning amplifier, the input voltage $0 \sim 3.3 \mathrm{~V}$ to $\mathrm{A} / \mathrm{D} \mathrm{DSP}$, and then through

the rear end of the clamp circuit to DSP the input end of the A/D.

\subsection{Design of zero crossing detection circuit}

The zero crossing detection plays two roles in the system: (1) the frequency of the grid voltage is measured; (2). These two are the necessary conditions for the system to track the grid voltage and the same frequency with the input current, so it is very important to design the zero crossing 
detection circuit.

The zero crossing capture circuit is used to capture the zero crossing point of the AC voltage of the network side, and the output signal is supplied to the CAP1 port of the DSP. The program of the software phase locked loop is used to calculate the frequency and phase information of the AC voltage by calculating the time interval between the two pulses. This circuit consists of Venturi filter circuit, zero crossing comparator circuit and voltage regulation circuit.

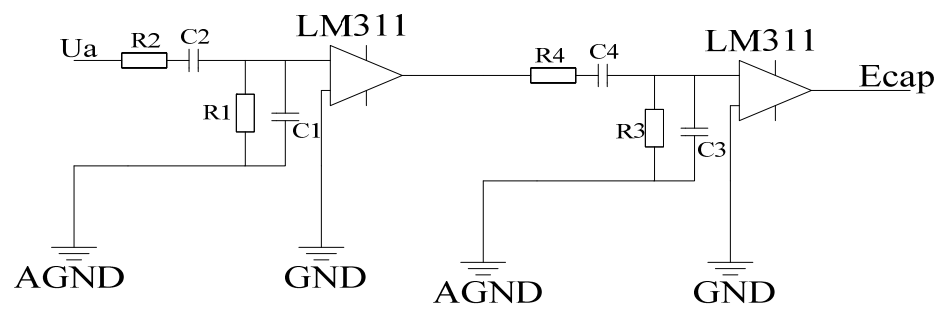

Fig. 6 The zero crossing detection circuit

The venturi filter circuit based on resonant principle, the minimum of the fundamental impedance and phase shift, including two parts of low-pass filter, high pass filter. R1 and C1, the composition of the low pass filter, low pass filter can filter out the harmonics of the input signal in the grid, the waveform is improved, but the phase had a lag, so the introduction of high pass filter R2, C2 compensation. As can be seen from the circuit, the high pass, low pass filter with identical topology, and $\mathrm{RC}$ phase symmetrical distribution, you can just ahead of the high pass filter phase delay offset low-pass filter. The filtered results after two times, not only to filter the harmonic sinusoidal, and no phase shift. In order to get a better phase signal R3, C3 and R4, C4 empathy.

\section{Conclusion}

Under the condition of uncontrolled rectifier A phase voltage and current waveforms as shown in Fig.7 (yellow for the current waveform, blue for the voltage).

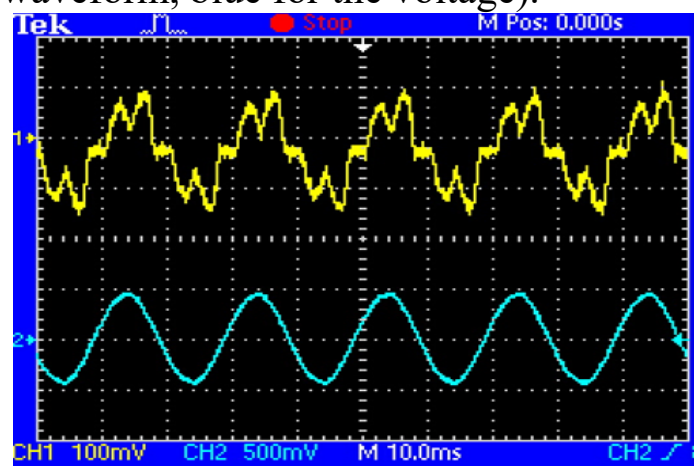

Fig.7 Under the condition of uncontrolled rectifier A phase voltage and current waveform

Under the condition of controlled rectifier A phase voltage and current waveform (yellow for the current waveform, blue for the voltage) as shown in Fig. 8 。

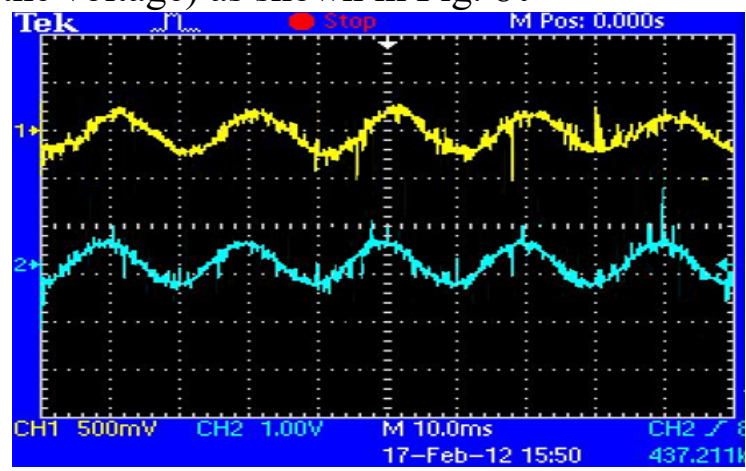

Fig. 8 Waveform of A phase voltage and current under controlled rectifier

By comparing Fig. 7 and Fig. 8, in the rectifier conditions, three-phase current waveform distortion, non sine wave, PWM rectifier mode, current waveform adjustment for sine wave, and 
voltage waveform phase, to achieve the desired purpose;

\section{References:}

[1] ZHANG Mingrong,LIU Fayou,WANG Delong et al.. Research and application of a simple infrared sulfur analyzer[J]. Shandong metallurgy. 200221 (10): 8-10.

[2]Yan Yi. Research on optical fiber methane monitoring technology based on ant colony algorithm [D] Master Thesis of Anhui University Of Science And Technology. Anhui.2013

[3]SU Hongsheng. Design of intelligent sulfur determination system based on P89C668 MCU[J]. Microcomputer and information. 2012 28(04): 38-41

[4]XIE En,WANG Zhaodong,WANG Xuewang. Research on fault diagnosis and fault tolerant control of electric vehicle motor drive system[J]. Journal of Jingdezhen Institute of technology..

[5]LUO Zhuowei,HU Zechun, SONG Yonghua, etc. Electric vehicle charging load calculation method[J]. Power system automation. 2011 35(14):36-40.

[6]Guo Dong Duan Lihua. Design of high performance permanent magnet synchronous motor speed control system based on TMS320C28346[J]Journal of Aviation Weapon, 2012, ( 5) : 20-24.

[7]Liliangyi. Design of permanent magnet synchronous motor speed control system based on DSPF2812 [J] Journal of Yangtze University, 2011, 8( 6) : 106-109. 